\title{
Saccade latency and warning signals: Effects of auditory and visual stimulus onset and offset
}

\author{
SUSAN M. ROSS and LEONARD E. ROSS \\ University of Wisconsin, Madison, Wisconsin 53706
}

\begin{abstract}
Previous studies have found that a nonspecific visual event occurring at the fovea 50-150 msec after the onset of a peripheral target delayed the initiation of the saccade to that target. The present studies replicated and extended this finding by studying the effects of both visual and auditory warning signals, by examining the effects of onset and offset warning on manual response latency, and by investigating the effects of presenting the warning events in the periphery of the visual field. The results indicated that the interfering effects occur with visual but not auditory stimuli, with saccades but not motor responses, and when the visual warning event occurs either foveally or in the subject's periphery. Implications for the processes involved are discussed.
\end{abstract}

When a nonspecific visual warning event precedes the onset of a peripheral target, the latency of the saccade to the target is shorter than when no warning event occurs (Becker, 1972; Cohen \& Ross, 1977, 1978; Saslow, 1967). Furthermore, Ross and Ross (1980) demonstrated that the onset, of fset, or change of a visual stimulus at the fixation point could facilitate the saccadic response when these warning events preceded the target, but that stimulus of fset was the most effective in reducing saccade latency. In addition, and most importantly for the present studies, it was found that when the onset warning event was presented after the occurrence of the target (a negative warning interval), the latency of the saccade was significantly longer than when the warning was the offset of a stimulus or when there was no warning. This result suggested that stimulus onset was not simply less effective than stimulus offset as a warning event, but that warning stimulus onset resulted in an active process or processes that interfered with or delayed the programming or execution of a saccade to a peripheral target. Thus, the interference was seen as reducing the warning effect when the onset warning event preceded the target by appreciable intervals (e.g., $300 \mathrm{msec}$ ) and also as delaying the saccade relative to the no-warning latency when the warning onset occurred from $150-50$ msec following target onset. No such delay was found when stimulus of fset was the warning event.

Ross and Ross (1980) suggested that the onset interference effect could be due to a number of factors. These included: (1) the possibility that, upon onset of the warning stimulus, the eye remained fixed to pro-

This research was supported by USPHS Grant HD08240. Requests for reprints may be addressed to either author, Psychology Department, University of Wisconsin, Madison, Wisconsin 53706. cess the new stimulus information, perhaps to permit its encoding and buffering to provide protection from suppression effects of subsequent fixations; (2) an interference due to the possible incompatibility between processes initiated by warning stimulus onset and segments of the programming sequence under way for the saccade to the peripheral target; or (3) the interfering effects resulting from the programming or execution of microsaccades that might be elicited by the onset of a new target appearing at the fixation point.

The investigation and clarification of the role of such factors in the onset interference effect would appear to depend upon a determination of certain of the effect's characteristics, such as whether it is specific to visual warning signals, whether it occurs with responses other than those involving the oculomotor system, and whether the presentation of the onset warning event at the fixation point is a necessary condition for its occurrence. With respect to the first question, it is possible that the superiority of of fset to onset as a warning event and the interfering effect of stimulus onset with negative warning intervals are related in some manner to stimulus onset-offset differences that are not necessarily limited to the visual modality. If this were found, the interpretation of the onset effect in terms of visual information intake efficiency, saccadic programming interference, and the effects of microsaccades could be largely irrelevant. Accordingly, Experiments $1 \mathrm{~A}$ and $1 \mathrm{~B}$ of the present study compared the effects of visual and auditory onset and of fset warning stimuli. Similarly, if the onset interference effect were found for response other than saccades, an interpretation involving programming mechanisms specific to the oculomotor system would be unnecessary. To investigate this possibility, Experiment 2 compared the effects of vi- 
sual stimulus onset and offset on manual responses. Finally, Experiment 3 compared the effects of visual warning onset and offset under peripheral presentation conditions to determine if presentation of stimulus onset at the point of fixation is necessary for the interference effect. Interference that occurs with foveal presentation of the stimulus could be due to information intake processes or to the competition that results from the programming or execution of small refixation eye movements. The finding of an interference effect with peripheral stimulus presentation would implicate only the latter type of factor-in this case, the interfering effects of some type of initial programming elicited by the warning signal on the ongoing programming of the saccade to the target.

\section{EXPERIMENTS 1A AND 1B}

\section{Method}

\section{Experiment 1A}

Subjects. The subjects were 32 undergraduate students ( 16 males and 16 females) enrolled in the introductory psychology course at the University of Wisconsin. All had 20/20 uncorrected near vision in both eyes, as measured by a Titmus Vision Tester.

Apparatus. The experiment was conducted in the two-room laboratory suite described in Ross and Ross (1980). The subject room was divided by a black curtain extending from floor to ceiling. A portion of the display screen, a Hewlett-Packard Model 1321 A display oscilloscope with a fast (P15) phosphor, was visible through a $2.5 \times 36.5 \mathrm{~cm}$ slot in a black panel located in the plane of the curtain. An AR-4X loudspeaker, located directly above the display scope, was used to present a continuous 45-dBA whitenoise background and $1,000-\mathrm{Hz}$ warning tones. The subject was seated in an ophthalmological examination chair equipped with an adjustable headrest. The chair was positioned so that the slot in the panel was at the subject's eye level at a viewing distance of $60 \mathrm{~cm}$. During the experiment, the subject room was dark except for a single $15-\mathrm{W}$ incandescent bulb located behind the curtain.

The stimulus-generating and response-recording equipment was located in an adjacent control room. White noise was produced by a Grason Stadler Model $901 \mathrm{~B}$ white-noise generator. The $1,000-\mathrm{Hz}$ warning tone, which was generated by a Hewlett-Packard Model 200 ABR audio oscillator, had a l-msec onset time as controlled by a Grason-Stadler Model 829E electronic switch. A laboratory computer generated the stimuli presented on the display screen and timed both visual and auditory stimuli. An intercom system permitted communication between the experimenter and the subject.

The subject's eye movements were detected using a Narco Biometric Eye Trac Model 200 eye movement monitor. Horizontal eye movements were recorded from the right eye, and vertical eye movements were recorded from the left eye. Permanent records of the unfiltered analog output of the Eye Trac were made using a Beckman R511A dynograph with a paper speed of $50 \mathrm{~mm} / \mathrm{sec}$. During each trial, the unfiltered Eye Trac output for the right eye (horizontal eye movements) was digitized by the laboratory computer, which identified the onset of the first saccade and determined its latency. At the conclusion of the trial, the trial conditions and saccade latency were printed, and the saccade, with the computer-determined saccade onset identified, was displayed on a monitor scope for the experimenter's inspection.

Design. A 2 by 2 design involving four groups of subjects was used to compare the effects of stimulus onset and offset as warning events. Two groups received visual warning signals (visualonset and visual-offset) and two received auditory warning signals (auditory-onset and auditory-offset). Each group was composed of four males and four females.
Warning interval, the interval between the onset of the warning stimulus and the onset of the target, was varied within subjects. Six warning interval conditions were employed-two in which the warning event occurred prior to the onset of the target, two in which the warning event occurred after the onset of the target, one in which the onset of warning and target was simultaneous, and a no-warning control condition in which no warning event occurred.

The two visual warning groups were run under the onset and offset conditions employed in the previous study (Ross \& Ross, 1980 ). For all conditions, four diagonal lines defined a 1.32-deg open fixation area (see Ross \& Ross, 1980). These lines remained on throughout the session. For the visual-onset group, the warning event was the appearance of a letter $O$ at the center of the fixation pattern. This letter, which subtended visual angles of $.5 \mathrm{deg}$ vertically and $.35 \mathrm{deg}$ horizontally, remained on until target of fset. For the visual-offset group, the letter $O$ was present at the center of the fixation area, and the offset of the letter served as the warning event. The $O$ reappeared within the fixation lines at the time of target of fset.

The warning event for the two auditory warning groups was the onset or offset of a $1,000-\mathrm{Hz} 70-\mathrm{dBA}$ tone, which was presented by a speaker located above the display screen. For the auditoryonset group, the onset of the tone served as the warning event, with the offset of the warning tone simultaneous with the of fset of the target. For the auditory-offset group, the tone was present during the intertrial interval, with its offset serving as the warning event. The tone remained off until target of fset.

All the subjects received 108 trials. The target letter $X$, which subtended a visual angle of $.5 \mathrm{deg}$ vertically and $.35 \mathrm{deg}$ horizontally, appeared $15 \mathrm{deg}$ to the left of the center of the screen on half of the trials and $15 \mathrm{deg}$ to the right on the other half. The target duration was $1 \mathrm{sec}$, and the occurrence of the left and right targets was arranged according to Fellows' (1967) sequences. All stimuli presented on the display screen had an intensity of 1 nit.

The 108 trials included 18 with each of six warning conditions: no-warning, $-300,-100,0,100$, and 300 msec of warning. On the no-warning trials, no warning event occurred. On -100 - and -300 -msec warning trials, the warning event occurred 100 or $300 \mathrm{msec}$, respectively, after the onset of the target. On 0-msec warning trials, the warning event was simultaneous with target onset, and on the 100 - and 300 -msec warning trials, the warning event preceded target onset by 100 or $300 \mathrm{msec}$, respectively. The warning conditions were arranged randomly within blocks of 36 trials, with the restrictions that, within each block, each warning condition occur three times with a left target and three times with a right target and that the same type of trial not occur twice in succession.

Procedure. Upon arriving at the laboratory, all the subjects received a vision test and were then seated in front of the display screen and were told that, from time to time, the letter $X$ would appear somewhere in the narrow window. The fixation stimulus was pointed out, and the subject was instructed to look at the $X$ as quickly as possible each time that it appeared. The subjects were asked to sit still and were encouraged to blink their eyes normally. The sensors were then fitted to the subject and adjusted as necessary, and the lights in the subject room, except for the 15-W lamp, were turned off. After a 2-min period, the eye-movement monitoring equipment was calibrated by having the subject look at various numbers in a horizontal five-number array. The procedure took about $5 \mathrm{~min}$, and trials began approximately $2 \mathrm{~min}$ after the disappearance of the calibration array.

Immediately prior to each trial, the subject's eye position was checked by the computer. If the subject's gaze was not within $1 \mathrm{deg}$ of the center of the fixation area or if the subject's eyes were closed, the trial was aborted and was attempted again approximately $3 \mathrm{sec}$ later. If the subject's gaze was still not within the fixation area, trials were halted briefly, and, if necessary, the Eye Trac sensors were recentered to compensate for changes in the subject's head position. Following each trial, the experimenter examined the tracing on the monitor oscilloscope and called for the next trial. This was followed by a computer-controlled pause of 
variable duration, causing the trials to occur at intervals of 6,10 , or $14 \mathrm{sec}$, with an average intertrial interval of $10 \mathrm{sec}$.

\section{Experiment 1B}

The purpose of this additional visual vs, auditory warning comparison was both to attempt to replicate the visual-auditory findings of Experiment $1 \mathrm{~A}$ and to determine if the onset-offset difference found in Experiment 1A could be contingent upon the presence of no-warning trials in the trial sequence. The subjects were 16 male and 16 female undergraduate students, enrolled in introductory psychology at the University of Wisconsin, who had $20 / 20$ uncorrected near vision in both eyes as measured by a Titmus Vision Tester. The same apparatus, method, and procedures were followed as in Experiment 1A except for the omission of the within-subjects no-warning condition. Each subject thus received 90 trials during the experimental session rather than the 108 in Experiment $1 \mathrm{~A}$.

\section{Results and Discussion}

Trials were deleted if a blink occurred between the onset of the warning signal and the target, if the first saccade began during the warning interval, if the first saccade was in the direction opposite to the location of the target, or if the first saccade was followed by a corrective saccade of more than $5 \mathrm{deg}$. In addition, trials were not used if the latency of the first saccade was less than $130 \mathrm{msec}$ or greater than $500 \mathrm{msec}$.

Each subject's mean response latency at each of the six warning intervals was determined separately for left and right targets. Since there were no sys- tematic differences between left and right latencies, the two were averaged for each subject. Group means of these latencies are shown in Figure 1 for both Experiments $1 \mathrm{~A}$ and $\mathbf{1 B}$.

If the interfering effects of an onset warning event reflect oculomotor processes involved in responses specific to a visual stimulus, then visual onset and offset warning should differentially affect saccade latency, but there should be no difference between auditory onset and offset warning. As Figure 1 indicates, such effects are present in both Experiments $1 \mathrm{~A}$ and $1 \mathrm{~B}$. The mean saccade latencies of Experiment $1 \mathrm{~A}$ were first analyzed by means of a fourfactor analysis of variance, with visual vs. auditory warning, onset vs. offset, and sex of subject as betweensubjects factors and the six warning intervals (no warning, $-300,-100,0$, and $300 \mathrm{msec}$ ) as a withinsubjects factor. The analysis resulted in a significant main effect of warning interval $[F(5,120)=114.82$, $\mathrm{p}<.001]$ and a significant interaction of modality and warning interval $[F(5,120)=8.99, p<.001]$. However, the result that is of primary interest in this analysis is a significant three-way interaction of warning modality, warning interval, and onset vs. offset $[F(5,120)=5.56, p<.001]$, which indicates that the functions relating onset and offset to warning interval for auditory signals were different from those for visual signals.

In order to evaluate the three-way interaction

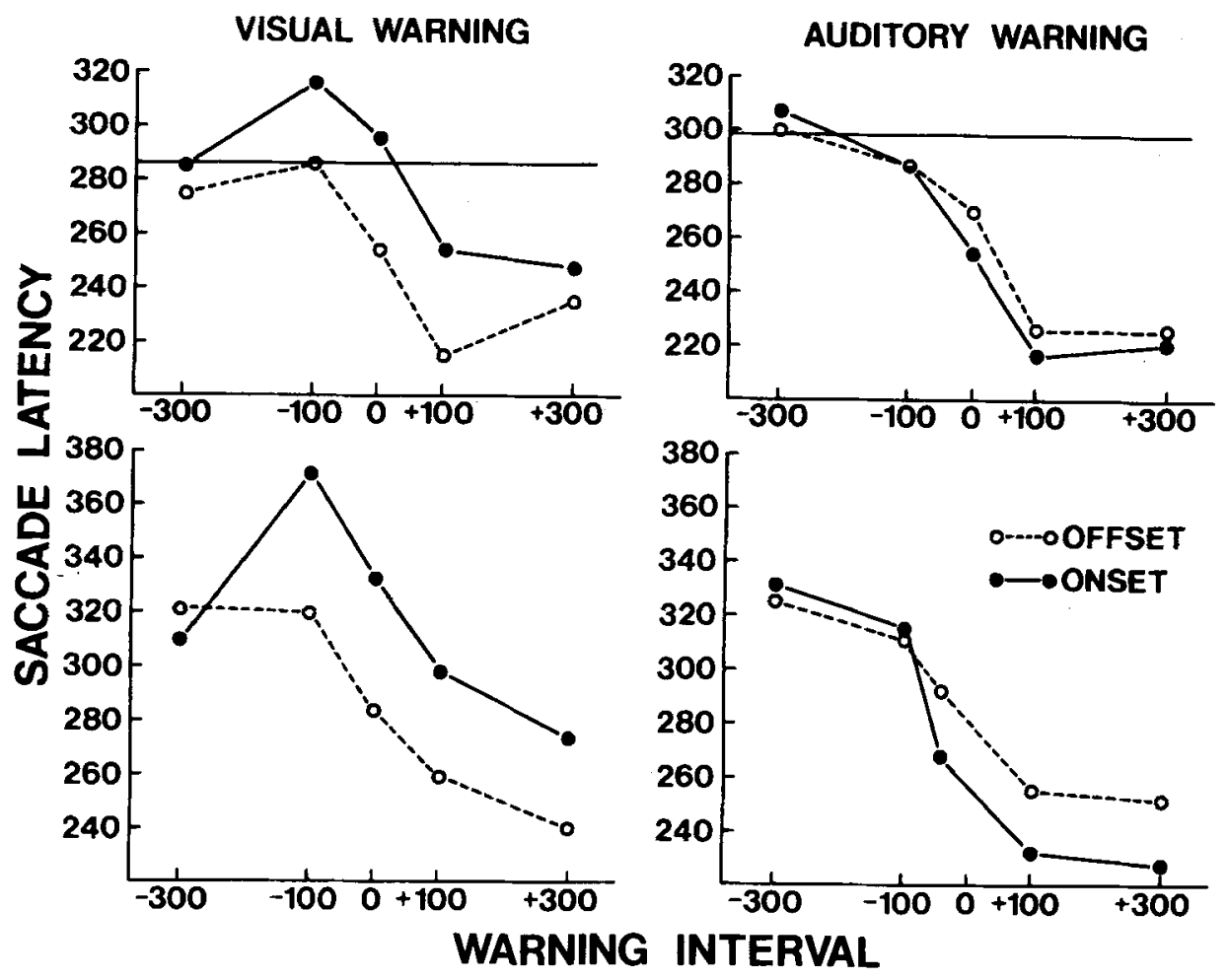

Figure 1. Mean saccade latency as a function of warning interval for visual and auditory onset and offset warning conditions. Results of Experiment $1 A$ are shown in the upper panels, and results of Experiment $1 B$ are shown in the lower panels. The horizontal lines represent the mean no-warning latencies. 
found in the overall analysis, two separate analyses were carried out, one involving only the visual warning data and the other involving only the auditory warning data: These analyses included onset vs. offset and sex of subject as between-subjects factors and warning interval as a within-subjects factor. Both analyses indicated a significant main effect of warning interval $[\mathrm{Fs}(5,60)=37.99$ and 91.35 for visual and auditory warning, respectively, $\mathrm{p}<.001$ for both]. The analysis for visual warning signals resulted in a significant interaction between warning interval and onset vs. offset $[F(5,60)=3.98, p<.005]$. This indicates that latencies under onset and offset warning conditions differed at some, but not all, warning intervals. The same interaction was not significant for the auditory warning subjects $[F(5,60)=1.98, p>.10]$, demonstrating that auditory onset and offset warning have similar effects at all of the warning intervals employed.

Subsequent $t$ tests were used to compare the mean latency at each of the warning intervals with the nowarning control latency in order to determine the warning intervals at which significant interference or facilitation was present. The visual-onset group's latency at $-100 \mathrm{msec}$ was significantly greater than its no-warning latency $[\mathrm{t}(60)=3.45, \mathrm{p}<.01]$, and the latencies at 100 and $300 \mathrm{msec}$ were significantly less than the no-warning latency $[\operatorname{ts}(60)=3.80$ and 4.63 , respectively, both ps $<.01$ ]. The visual-offset group's latencies were significantly below its no-warning latency at warning intervals of 0,100 , and $300 \mathrm{msec}$ $[\operatorname{ts}(60)=3.68,9.40$, and 5.96, respectively, all ps $<.01]$. These results replicate the differences between visualonset and visual-offset warning found by Ross and Ross (1980). While both reduce saccade latency when the warning interval is $100 \mathrm{msec}$ or greater, only visual-onset warning has an interfering effect at the -100 -msec warning interval.

As in the case of the visual-offset warning data, both auditory-onset and auditory-offset warnings facilitated saccadic responding at warning intervals of 0,100 , and $300 \mathrm{msec}[\operatorname{ts}(60)=6.77,14.13$, and 13.98 for $0-, 100-$, and $300-\mathrm{msec}$ warnings, respectively, $p<.01$ for all three]. However, in contrast with the results for the visual warning groups, there was no evidence that auditory-onset warning exerted an interfering effect on saccadic responding when the auditory stimulus onset occurred after target onset. This outcome indicates that the interference effects seen in the visual-onset data reflect processes specifically associated with the visual modality, presumably either in terms of greater processing requirements associated with the onset of a visual stimulus or in terms of the interfering effects of these processes on the programming of subsequent saccades.

A very similar pattern of differences is seen in the results of Experiment $1 B$. The overall analysis of variance on mean latencies also resulted in a significant three-way interaction of modality, onset vs. offset, and warning interval $[F(4,96)=8.36, p<.001]$. The two subsequent analyses of variance resulted in a significant interaction of the onset-offset variable and warning interval $[F(4,48)=7.42, p<.001]$ for the visual warning groups and a significant main effect of warning interval for the auditory warning groups $[F(4,48)=71.30, p<.001]$. Subsequent planned $t$ tests found significant differences between visual-onset and visual-offset at all warning intervals except the $-300-\mathrm{msec}$ value. Thus, the data of Experiment 1B fully support the conclusion of Experiment $1 \mathrm{~A}$ that the onset-offset effects found in that experiment and by Ross and Ross (1980) are specific to visual warning.

\section{EXPERIMENT 2}

Experiment 2 was carried out to determine if the visual onset-offset warning differences found in Experiments $1 \mathrm{~A}$ and $1 \mathrm{~B}$ would also occur with responses other than saccades. Accordingly, Experiment 2 employed the same visual warning onset and offset stimulus conditions as were used in Experiments $1 \mathrm{~A}$ and $1 \mathrm{~B}$, but the subjects were given the task of moving a lever to the right or left upon the target's appearance on either side.

\section{Method}

\section{Subjects}

The subjects were 16 undergraduate students $(8$ males and $8 \mathrm{fe}-$ males) enrolled in the introductory psychology course at the University of Wisconsin. All had 20/20 uncorrected near vision in both eyes, as measured by a Titmus Vision Tester.

\section{Apparatus \\ The experiment was conducted using the apparatus described in Experiment 1. In addition, a vertical response lever was fixed to the end of the armrest of the subject's chair on the subject's pre- ferred side. The lever, which was $13 \mathrm{~cm}$ in length, could be moved $15 \mathrm{deg}$ to the left and right of the center vertical position and was lightly spring-loaded to return to the vertical when released by the subject. The lever was directly attached to a potentiometer, and resistance changes, indicating lever position, were recorded on the Beckman Dynograph along with the Eye Trac output and trial event markers.}

\section{Design and Procedure}

The subjects were divided into two groups of four males and four females each. The visual-onset and visual-offset stimulus conditions employed were the same as those described in Experiment 1. The set of warning intervals and the sequence of trials were the same as those used in Experiment 1A.

The procedure was the same as that used in Experiment 1, with the exception that subjects were instructed to hold the lever and to move it as quickly as possible on every trial to the side on which the $\mathbf{X}$ appeared. Looking at the $\mathbf{X}$ was not mentioned in the instructions.

\section{Results and Discussion}

Manual response latency was measured to the nearest $10 \mathrm{msec}$ from the dynograph recordings. 
Trials were discarded if the subject initially moved the lever in the direction opposite to the target or if the latency of response was less than $130 \mathrm{msec}$ or greater than 1,000 msec. Each subject's average response latency under each of the six warning conditions was determined separately for left and right targets. Since no systematic differences were found between left and right latencies, the two latencies were averaged for each subject. Group means of these latencies are shown in Figure 2.

Mean latencies were analyzed by means of a threefactor analysis of variance with sex of subject and onset vs. offset as between-subjects factors and the six warning intervals as a within-subjects factor. The analysis indicated a significant main effect of warning interval $[F(5,6)=60.18, p<.001]$ but no onsetoffset effect $[F(1,12)=.01]$ and no interaction of onset-offset and warning interval $[F(5,60)=.86]$. Subsequent $t$ tests comparing the average latency for the no-warning control condition with the average latencies at each of the other warning intervals indicated significantly shorter latencies at warning intervals of 0,100 , and $300 \mathrm{msec}[\operatorname{ts}(60)=2.72,9.67$, and 13.19 , respectively, $p<.01$ for all three comparisons].

The failure to find onset-offset warning differences with visual stimuli and a manual response, together with a similar lack of an onset-offset effect with auditory warning events, strongly suggests that the offset superiority is specific to visual warning stimuli and oculomotor responses. In addition, the similarity of the offset warning effect under all conditions is striking. Comparison of the offset warning effect at different warning intervals shows almost identical functions for both manual and oculomotor responses when visual stimuli were used as warning events and for oculomotor responses when auditory warning events were employed. In no case were of fset warning saccade latencies found that were longer than those obtained under no-warning control condi-

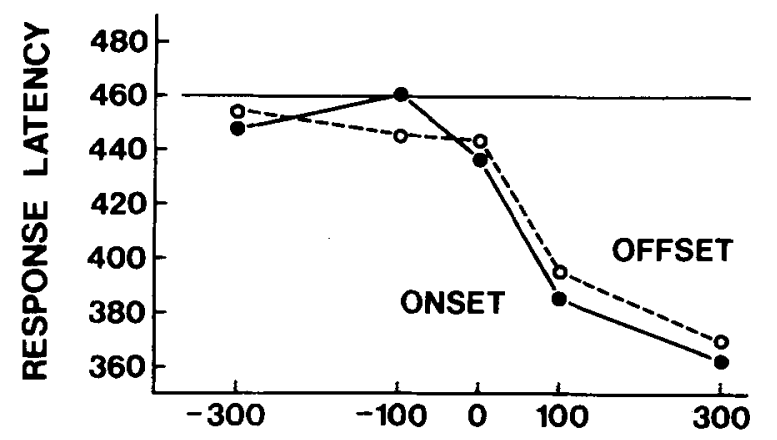

WARNING INTERVAL

Figure 2. Mean manual response latency as a function of warning interval for visual onset and offset warning conditions. The horizontal line represents the mean no-warning latency. tions, and, in each instance, offset warning facilitated saccadic responding when the warning event was simultaneous with the target or preceded it by 100 or $300 \mathrm{msec}$. In contrast, the combination of visual onset warning signals and oculomotor responding has consistently resulted in negative warning-interval saccade latencies that were longer than those found under no-warning conditions and in positive warning interval effects that were somewhat less than those found with visual offset.

\section{EXPERIMENT 3}

All of the previous experiments' visual onset and offset warning stimuli were presented at the subjects' fixation point. Experiment 3 investigated the effects of onset and offset visual warning stimuli when the stimulus events occurred in the peripheral visual field rather than at the point of fixation. Interest was in whether the onset-offset differences previously demonstrated would also occur under peripheral presentation conditions. Onset and offset warning stimuli were presented in pairs symmetrically above and below or to the left and right of the fixation point in order to reduce the frequency of saccades to the warning stimuli below the number that might have been made to a single warning stimulus. It was thought that the vertical and horizontal warning stimulus conditions might differ in effectiveness as warning events or in the degree to which they would elicit saccades prior to the presentation of the target.

\section{Method}

\section{Subjects}

Subjects were 32 undergraduate students (16 males and $16 \mathrm{fe}$ males) enrolled in the introductory psychology course at the University of Wisconsin. All had 20/20 uncorrected near vision in both eyes, as measured by a Titmus Vision Tester.

\section{Apparatus}

The experiment was conducted using the apparatus described in Experiment 1, with the exception that the slot in the black panel behind which the display screen was located was enlarged to a size of $35.5 \times 15.3 \mathrm{~cm}$ in order to accommodate stimuli above and below the previous stimulus positions.

\section{Design and Procedure}

The subjects were divided into four groups of four males and four females each. Two of the groups were run under conditions similar to visual-onset described in Experiment 1, except that instead of a single warning symbol appearing at the center of the fixation lines, the warning event consisted of the simultaneous onset of two 0s. For one group (vertical-onset), the 0s appeared $5.5 \mathrm{deg}$ above and below the center of the fixation pattern, and, for the other group (horizontal-onset), the two 0s appeared 5.5 deg to the left and right of the center of the fixation pattern. The remaining two groups were run under offset warning conditions that were identical to the onset conditions, except that the warning event was the offset of the 0s, which were otherwise present from targets offset to the warning event.

The set of warning intervals and trial sequences was the same as that used in Experiment 1. Procedures and instructions to the sub- 
ject were the same as those used in Experiment 1, with the exception that two more points, $5.5 \mathrm{deg}$ above and below the central horizontal array, were added to the array of digits used to calibrate the eye-movement monitoring equipment.

\section{Results and Discussion}

Each subject's record was examined and scored as described in Experiment 1. In addition, trials on which the subject looked at either of the warning stimuli prior to looking at the target were identified and deleted from the data for separate treatment. Criteria for identifying these trials were an upward or downward saccade of $3 \mathrm{deg}$ or more for the vertical onset and offset groups and a leftward or rightward saccade of more than $3 \mathrm{deg}$ but less than $8 \mathrm{deg}$ for subjects in the horizontal onset and offset groups. Each subject's average response latency was determined by separately averaging latencies for left and right targets at each warning interval. Since there were no systematic differences between response latencies to left and right targets, they were combined.

Examination of group averages of saccade latencies indicated substantial differences among the groups in response latency on the no-warning trials, with values ranging from $278 \mathrm{msec}$ for the verticalonset group to $318 \mathrm{msec}$ for the hroizontal-offset group. Given these differences, each subject's data were converted to deviation latencies by subtracting the subject's mean no-warning latency from the response latency at each of the five warning intervals. Group means of these deviation latencies are shown in Figure 3. Note that the horizontal-offset group point for a warning interval of $300 \mathrm{msec}$ is not connected to the other data points in order to reflect the fact that four subjects in that group responded to both the warning stimulus and target on every 300 msec warning trial and therefore could not be included in the average.

From Figure 3, it can be seen that the onset-of fset warning effect still occurred when warning stimuli were presented peripherally in either the horizontal or vertical plane. The similarity of these functions to those obtained with foveally presented warning stimuli demonstrates that, contrary to expectations, the interfering effect of onset warning is not limited to foveal presentation. The analysis of these onset and offset effects-which included warning location (vertical vs. horizontal), onset vs. offset, and sex of subject as between-subjects factors and warning interval as a within-subjects factor-confirmed the similarity of these findings to previous data. The analysis yielded significant main effects of onset vs. offset $[F(1,24)=$ $10.10, \mathrm{p}<.005]$, warning interval $[F(4,92)=83.58$, $\mathrm{p}<.001]$, and the interaction between these two variables $[F(4,92)=17.15, p<.001]$. This interaction indicates that deviation latencies under onset and of $f$ set warning conditions differed at some, but not all, warning intervals. Subequent $t$ tests found latencies
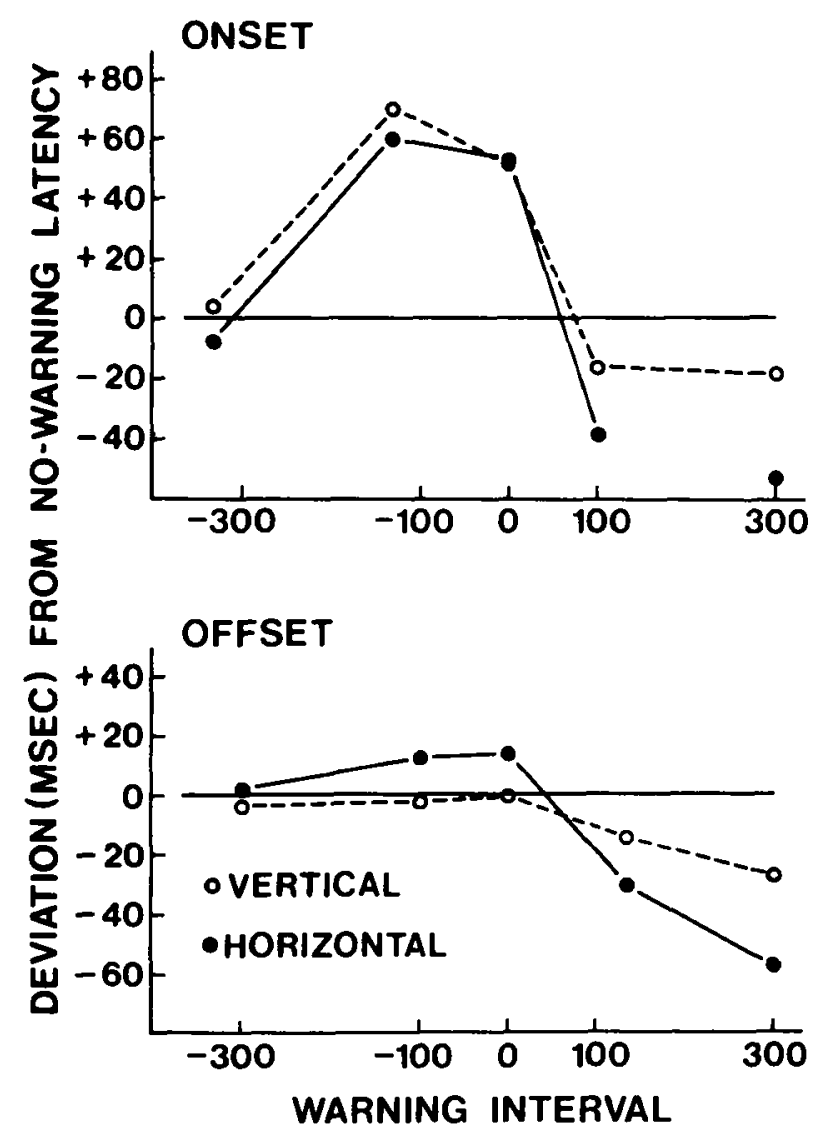

Figure 3. Mean deviation latencies as a function of warning interval, warning event location, and onset vs. of fset warning.

under onset warning conditions to be significantly greater than of fset warning latencies at warning intervals of -100 and $0 \mathrm{msec}[\operatorname{ts}(92)=8.39$ and 6.38 , respectively, $\mathrm{p}<.001$ for both]. Thus, the peripheral visual onset and offset warning signals employed in this experiment had essentially the same interfering effect on saccade latency as did visual onset and offset warning signals presented at the fixation point in Experiment 1.

The similarity of the results for foveal and peripheral presentation of the warning event does not permit one to conclude that the processes involved are the same, however. The interfering effects of a foveal warning stimulus onset could reflect processes that delay a change in fixation until information from the new event is encoded (e.g., Potter, 1976), while the effects of peripheral warning onset could primarily reflect competition between the initial stages of programming for a saccade to the warning stimulus onset and the ongoing programming for the saccade to the target. Alternatively, the foveal effect could reflect saccadic interference due to fixation microsaccades or their programming. The fact that the interference occurs when warning onset follows target onset by $100 \mathrm{msec}$ with both foveal and peripheral warning suggests, however, that, regardless of the similar- 
ity or differences of the processes underlying the effect, their temporal characteristics and their delaying effect on the saccade to the target are quite similar.

One aspect of the horizontal and vertical peripheral warning effect did differ from that of foveal warning in that $t$ tests found no differences between onset and offset at warning intervals of 100 and $300 \mathrm{msec}$. This is in contrast with the foveal warning data from Experiment 1 and the earlier study (Ross \& Ross, 1980) that showed a significant onset-offset difference at these warning intervals. This apparent interaction between the onset-offset warning variable and foveal vs. peripheral warning event location lends some support to the notion that different processes are responsible for the foveal and peripheral effects of onset warning. While an onset at the point of fixation may elicit processes that strengthen fixation or inhibit eye movements away from the fixation area and thus lead to of fset superiority, an onset outside the fovea could "release" the eye from fixation in much the same way as is assumed for foveal offset so that no onset-offset differences in facilitation of the saccadic response would be expected.

It might be argued that the interaction between the onset-offset and warning interval variables reflects differences in the latency of the perceptual response to onsets and offsets in the fovea and the periphery. For example, there is evidence (Hansteen, 1971; Lewis, Dunlap, \& Matteson, 1972; Walsh, 1973) that the perceptual latency to a foveal onset is about $40 \mathrm{msec}$ longer than the latency to a foveal of fset, while the corresponding difference at the periphery is smaller and less consistent. However, although the pattern of results is consistent with such perceptual latency differences, it seems doubtful that such a factor could account for the interaction. Since the target in the present studies was always an onset in the periphery, regardless of the type or location of the warning, the perceptual latency differences could affect only detection of the warning event. In addition, the differences in perceptual latency are small relative to the duration of the warning signal, especially at the 300msec warning interval. Thus, while perceptual latency differences might possibly contribute to the interaction between type of warning and location, such an effect, if it existed, would probably be quite small.

Warning signal position (i.e., vertical vs. horizontal) had no significant effect $[F(1,24)=1.90]$ and did not interact with the onset-offset variable $[F(1,24)=$ $1.20]$. There was a significant interaction between position and warning interval $[F(4,92)=5.71, p<$ $.001]$, which indicates that vertical and horizontal warning signals were not equally effective at all warning intervals. Subsequent $t$ tests showed no difference between vertical and horizontal warning at warning intervals of $-300,-100$, and $0 \mathrm{msec}$, where the onset-offset differences appeared $[\operatorname{ts}(92)=.42, .30$, and 1.16, respectively]. However, when the warning event preceded the target by 100 or $300 \mathrm{msec}$, saccade latencies were significantly shorter with horizontal warning signals than with vertical warning signals $[\mathrm{t}(92)=2.74, \mathrm{p}<.01$, and $\mathrm{t}(92)=4.68, \mathrm{p}<.001$, respectively]. Since the target always appeared in the horizontal meridian, these results raise the possibility that a warning event occurring in the same meridian as the subsequent target is more effective than a warning event occurring in the orthogonal meridian-in this case, the vertical. It is possible that a horizontal warning event initiated the programming of a horizontal saccade, regardless of the instructions regarding the target and the subject's intentions not to look at the warning events, and that there was some savings in the subsequent programming of the horizontal saccade to the target. A similar conclusion regarding the possibility of such facilitating and interfering effects was reached by Hou and Fender (1979) in a study in which double-step targets were presented, with the subject instructed to follow their occurrence as quickly as possible. When the targets were presented in rapid succession, the subjects apparently canceled saccades to the first target and reprogrammed their saccades to fixate the second target only. When the reprogramming involved the cancellation of a saccade in one meridian and the reprogramming of a saccade in the orthogonal meridian, reaction times were longer than reaction times to single targets. Hou and Fender hypothesized that when a saccade is canceled, the results of saccade programming processes are retained in a buffer, and if the direction of subsequent saccade is different from that of the canceled saccade, the contents of the buffer must be erased before further programming can proceed, thus increasing saccade latency. If the direction of the second saccade is similar to the canceled saccade, the saccade computations need only be modified, thus reducing saccade latency. An alternative possibility is that the shorter latency of saccades on horizontal warning trials could reflect the fact that more horizontal trials involving double responses were deleted from the data and that the remaining horizontal warning trials were less likely to have interfering effects from competing programming. Thus, the horizontal-vertical latency difference could have resulted from a greater propensity for interfering programming in the horizontal plane to result in double responses.

The percentage of trials on which the subjects made a saccade to the warning event prior to looking at the target was determined for each group. Such double saccade responses were rare in the two of fset warning groups (less than $2 \%$ ), but somewhat more frequent in the two onset groups, as can be seen in Figure 4, in which double responses are shown for trial blocks as a function of warning interval. As the figure suggests, saccades to the warning event were more frequent with horizontal than with vertical 


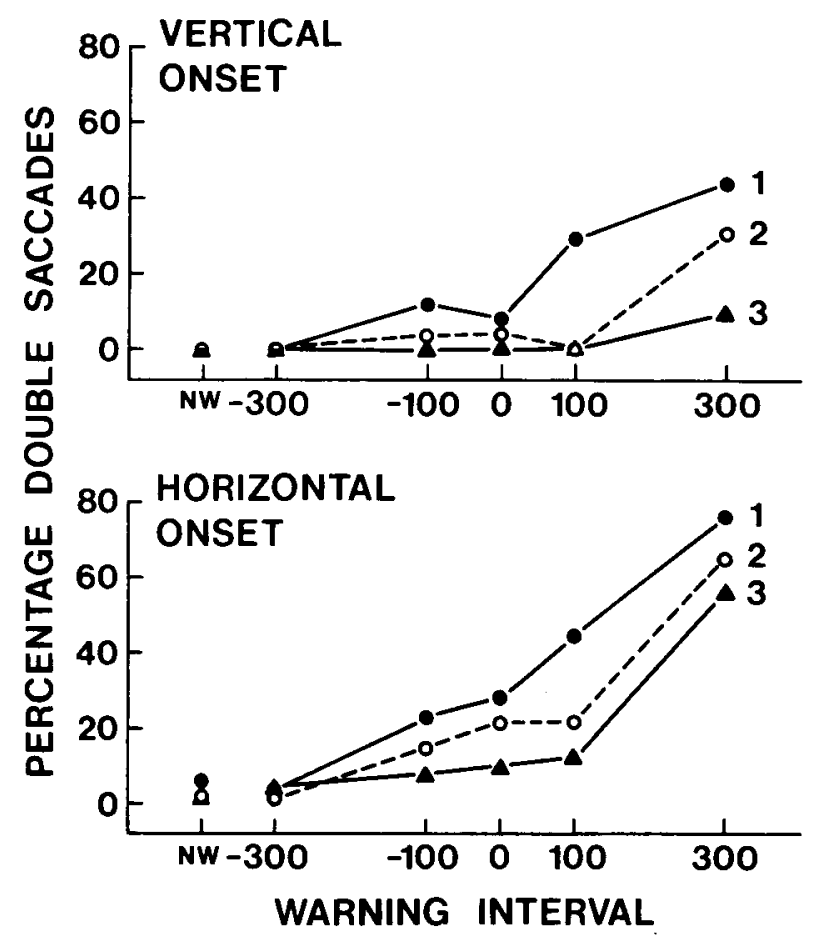

Figure 4. Percentage of trials on which the subjects made a saccade to both the warning event and the target. Percentages are shown for three trial blocks of 36 trials each as a function of warning interval (NW = no-warning).

warning conditions $[F(1,12)=8.60, p<.025]$, and their frequency varied with warning interval $[F(5,60)$ $=35.14, \mathrm{p}<.001]$ and decreased across trial blocks $[F(2,24)=11.23, \mathrm{p}<.005]$.

Although the double saccades occurred primarily when the warning event preceded the target and not at the intervals at which the onset warning effect was present, the fact that the frequency of double saccades changed across trial blocks suggested the possibility that the effect of onset warning at warning intervals of -100 and $0 \mathrm{msec}$ might show similar variation. In order to determine whether the interference effect varied as a function of trial blocks (i.e., as the frequency of double responses changed), mean deviation latencies were computed for each block of trials. The results are shown in Figure 5, in which it is evident that the shapes of the curves change little as a function of trial block and that there are only small differences between vertical and horizontal onset groups. These observations were confirmed by a four-factor analysis of variance (warning position, warning interval, trial blocks, and sex of subject as factors), which yielded only a significant main effect of warning interval $[F(4,48)=68.77, p<.001]$. Thus, there seems to be no relationship between the interfering effect of onset warning and the changing frequency of double saccades across trial blocks.

\section{GENERAL DISCUSSION}

The results of these experiments demonstrate both the generality of the facilitating effect of a nonspecific warning or alerting event preceding a target stimulus and the specificity of the interfering effect of an onset warning event that follows target onset. The general effects of a preceding warning event can be seen in all three studies, in which response latency was shorter when the nonspecific warning preceded the target than when no warning event occurred. This held for both visual and auditory warning events when the response was a saccade and for manual responses under visual warning conditions.

Of greater importance, however, is the fact that the three studies strongly suggest that the interfering effect seen when an onset warning event occurs after the target is specific to the visual modality and the oculomotor system. The results of Experiment 1 indicated that the interference effect is not seen with
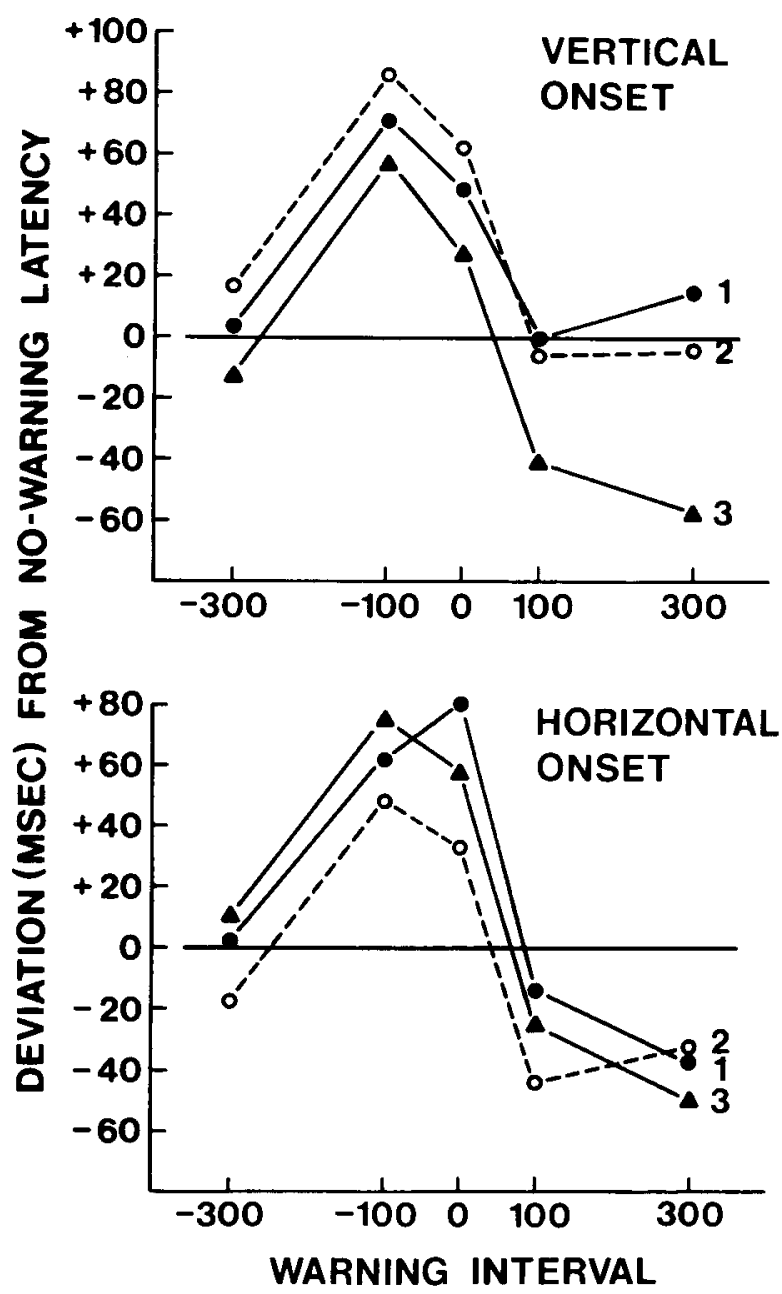

Figure 5. Mean deviation latencies for three blocks of 36 trials as a function of warning interval. 
auditory warning events, and the results of Experiment 2 indicated that the effect does not occur with visual stimuli when the response is a hand movement rather than an eye movement. However, as indicated by the results of Experiment 3, it is not necessary for the visual onset to occur at the point of fixation, as demonstrated by the fact that the interfering effect was present even when the onset warning occurred 5.5 deg away from the fovea.

These results suggest interpretations based on oculomotor programming mechanisms. There seem to be at least two obvious possible interpretations for the interfering effects of foveal onset following the target and one such interpretation for the peripheral onset's interfering effect. The first interpretation involves primarily oculomotor processes. The onset of a stimulus at the fovea could elicit microsaccades associated with fixation of the new stimulus, and either the programming or the actual execution of these microsaccades, which would not have been detected in these studies, could interfere with the programming or execution of the saccade to the target. The second interpretation for the interfering effects of a foveal onset involves somewhat different processes. Potter and others (e.g., Potter, 1976; Potter \& Levy, 1969; Senders, 1976) have suggested that the eye remains relatively fixed at the point at which information is being presented rapidly. The processes assumed to underlie this effect include the encoding of the incoming information and the buffering of the new information in order to protect it from the suppression effects of subsequent saccades. If the visual onset warning event occurring at the fovea initiated such processes, inhibition of the saccade to the target might be expected, even though the programming of that saccade was already under way when the foveal onset occurred.

The most obvious interpretation of the interfering effects of peripheral onset warning involves mainly oculomotor processes-specifically, the competition between the programming of the saccade to the target and some initial programming for a saccade to the location of the warning event. Even if a saccade to a warning stimulus is not executed, the appearance of a new stimulus may elicit some program- ming or, conceivably, an attentional process that interferes with the ongoing programming of the saccade to the target.

Future research will be necessary to determine which of the various processes is related to the interference effect, the conditions under which it occurs, and the degree to which the effect might be related to other stimulus and attentional factors.

\section{REFERENCES}

BECKER, W. The control of eye movements in the saccadic system. In J. Dichgans \& E. Bizzi (Eds.), Cerebral control of eye movements and motion perception. Basel, Switzerland: Karger, 1972.

Cohen, M. E., \& Ross, L. E. Saccadic latency in children and adults: Effects of warning interval and target eccentricity. Journal of Experimental Child Psychology, 1977, 23, 539-549.

Cohen, M. E., \& Ross, L. E. Latency and accuracy characteristics of saccades and corrective saccades in children and adults. Journal of Experimental Child Psychology, 1978, 26, 517-527.

Fellows, B. J. Chance stimulus sequences for discrimination tasks. Psychological Bulletin, 1967, 67, 87-92.

Hansteen, R. W. Visual latency as a function of stimulus onset, offset, and background luminance. Journal of the Optical Society of America, 1971, 61, 1190-1195.

Hou, R. L., \& Fender, D. H. Processing of direction and magnitude by the saccadic eye-movement system. Vision Research, $1979,19,1421-1426$.

Lewis, J. H., Dunlap, W. P., \& Matteson, H. H. Perceptual latency as a function of stimulus onset and offset and retinal location. Vision Research, 1972, 12, 1725-1731.

PotTer, M. C. Short-term conceptual memory for pictures. Journal of Experimental Psychology: Human Learning and Memory, 1976, 2, 509-522.

Potte R, M. C., \& Levy, E. I. Recognition memory for a rapid sequence of pictures. Journal of Experimental Psychology, 1969, 81, 10-15.

Ross, L. E., \& Ross, S. M. Saccade latency and warning signals: Stimulus onset, offset, and change as warning events. Perception \& Psychophysics, 1980, 27, 251-257.

Saslow, M. G. Effects of components of displacement-step stimuli upon latency for saccadic eye movement. Journal of the Optical Society of America, 1967, 57, 1024-1029.

Senders, J. W. Speculations and notions. In R. A. Monty \& J. W. Senders (Eds.), Eye movements and psychological processes. Hillsdale, N.J: Erlbaum, 1976.

WALSH, T. Visual onset and of fset latencies. Quarterly Journal of Experimental Psychology, 1973, 25, 154-162.

(Received for publication August 22, 1980; revision accepted January 12, 1981.) 